\title{
A New Method of Online Measurement Viscosity of Paint dissolved liquid for Enameled Wire
}

\author{
Weixiong $\mathrm{Xu}^{1,}$, Adi yang ${ }^{1, \mathrm{~b}}$, Wenzhen Zhang ${ }^{2, \mathrm{c}}$ \\ ${ }^{1}$ Palletizing Robots Engineering Center of universities in fujian province, China \\ ${ }^{2}$ Huaqiao University, China \\ a2308798018@qq.com, b380858090@qq.com, czwz@hqu.edu.cn
}

Keywords: Microcontroller, Buzzer, Viscosity.

\begin{abstract}
The viscosity of paint dissolved liquid for enameled Wire is key that manufacture enameled wire, paint dissolved liquid is volatilization and change every moment, this paper presents a new method for real-time measurement of the viscosity of paint dissolved liquid for enameled wire, the new method is by piezoelectric effect and inverse piezoelectric effect, and supported by PIC16F877 single-chip microcomputer, the viscosity measurement and the real-time change of the viscosity of paint dissolved liquid, in order to produce the high quality of the enameled wire.
\end{abstract}

\section{Introduction}

In the production process of enameled wire, the viscosity of paint dissolved liquid has a critical effect on the quality of enameled wire, the painting of the surface layer of the enameled wire adopts a thin-paint-coating craft process. The enameled wire is usually coated with a thin layer of paint and dried in the drying stove, after this another thin layer of paint is coated and dried in the drying stove again, so repeatedly, however, if the insulating paint viscosity is not well controlled, the coat of the enameled wire will be uneven, which leads to be poor or scrapped.

The traditional method to measure the viscosity of paint dissolved liquid is timed sampling using the dosage cup, taking the time that quantitative paint consumes passing through the custom funnel as the relative viscosity .However, due to the continuous production process of enameled wire, the interval sampling will cause a big delay, the viscosity of paint dissolved liquid can't be adjusted in real time. Besides, the various cylinders of the thin paint coating process are open, due to changes in ambient temperature, humidity and other factors, such as temperature, dry climate, paint solvents are likely to be volatile, so the measurement of the enameled wire paint viscosity is an important part of enameled wire production.

Hao Pan [1] proposed a new method to measure Liquid viscosity coefficient, Li Ying [2] pointed Online Jisi cut the throat diameter of the order of several tens of enameled wire paint process, due to the natural evaporation of the solvent, the viscosity of the paint will increase gradually which causes unevenness of the enameled wire coating. To solve this problem, the author puts forward a new method to measure the enameled wire viscosity based on microcontroller and the buzzer.

\section{The Theory of Viscosity Measurement}

Using the sensor characteristics of the buzzer, we can get the viscosity coefficient by measuring the resonance frequency of the forced vibration in the liquid. The specific theory as follows:

If the quality is $\mathrm{m}$, the motion equation of the buzzer's forced movement in the liquid is as follows:

$$
\mathrm{m}\left[\frac{d^{2} x}{d t^{2}}+2 \beta \frac{d x}{d t}+\omega_{0}{ }^{2} x\right]=m f_{0} \sin \omega t
$$


Among this, $\beta$ is the damping coefficient, $\omega_{0}$ is the system natural frequency, $\operatorname{mf}_{0} \sin \omega t$ is a fixed frequency periodic external force. The solution of the equation obtained is as follows:

$$
x=x_{0} \sin (\omega t+\phi)
$$

The derivative of $\mathrm{x}$ :

$$
\begin{aligned}
& \frac{d x}{d t}=\omega x_{0} \cos (\omega t+\phi) \\
& \frac{d^{2} x}{d t^{2}}=-\omega x_{0} \sin (\omega t+\phi)
\end{aligned}
$$

The motion Eq. (1) becomes as:

$$
\left(\omega_{0}^{2}-\omega^{2}\right) x_{0} \sin (\omega t+\phi)+\beta \omega x_{0} \cos (\omega t+\phi)=f_{0} x_{0} \sin (\omega t)
$$

Through a series of derivation, the amplitude of forced vibration is:

$$
x_{0}=\frac{f_{0}}{\sqrt{\left(\omega_{0}^{2}-\omega^{2}\right)^{2}+(\beta \omega)^{2}}}
$$

In the case of resonance, the periodic force $\omega$ is equal to the natural frequencies of the system. In fact, because of the influence of the damping coefficient of $\beta$, it is not equal. The derivative of Eq. (5) is:

$$
\frac{d}{d \omega}\left[\left(\omega_{0}^{2}-\omega^{2}\right)^{2}+(\beta \omega)^{2}\right] \omega=0
$$

then we can get Eq. (7) from Eq. (6):

$$
\omega_{m}=\sqrt{\omega_{0}^{2}-2 \beta^{2}}
$$

By comparing the natural frequency of the system and its impressed frequency, we can get the damping coefficient $\beta$. The damping coefficient $\beta$ is proportional to the viscous coefficient $\eta$ :

$$
\eta=k \beta
$$

\section{The Experiment of Viscosity Measurement}

After connecting to the signal generator, the Piezoelectric buzzer will vibrate. Put the Piezoelectric buzzer in the measured enameled wire paint, we can get the frequency and voltage signals through PIC16F877 microcontroller, the thicker the enameled wire paint is, the lower the frequency and voltage signals will be. If we change the frequency of the frequency generator, there will be a voltage change on the oscilloscope. In generally, the output power of the signal generator is constant, when the minimum voltage occurs, the Piezoelectric buzzer will fall in the notch state, and the frequency is the Piezoelectric buzzer's resonant frequency.

As the temperature has a great influence in the output voltage and frequency of the Piezoelectric buzzer, we must heat the enameled wire paint bucket appropriately and take temperature control to make a constant temperature.

Place two pieces of Piezoelectric buzzers in the enameled wire paint bucket, the two pieces of Piezoelectric buzzer chips are placed in relative position, the distance between each other must be at least $20 \mathrm{~mm}$, one of them is connected to the pulse output of PIC16F877 microcontroller, and the other is connected to the input port of the PIC16F877 microcontroller which will turn the frequency signal into a level signal, and then through the A / D conversion, we can get the real-time wire 
coating concentration changes through the calculation and the relative comparison by the microcontroller.

Hardware Circuit. Piezoelectric buzzer 1 has an inverse piezoelectric effect, which will convert the amplified pulse signal comes from microcontroller into mechanical vibrations, Piezoelectric buzzer 2 has a positive effect, which will converts the mechanical vibration signal into electrical signal. The microcontroller will turn the electrical signal which receives form A/D converter into digital signal, after the calculation and comparison, the touch screen will display the viscosity of paint of paint barrels according to the calculation result of the microcontroller.

The temperature has an important effect on the piezoelectric effect, so we need to heat the entire paint bucket properly in order to keep the control temperature as a constant number and promote to mix and react between polyurethane paint and solvent fully.

Thermal resistance temperature signals are transferred to the A/D converter after amplified by the circuit in the microcontroller, then controlled the silicon controlled single-phase circuit to heat the lacquer bucket(heating by heating rods and prohibiting open flames). The viscosity measurement circuit is shown in Fig. 1.

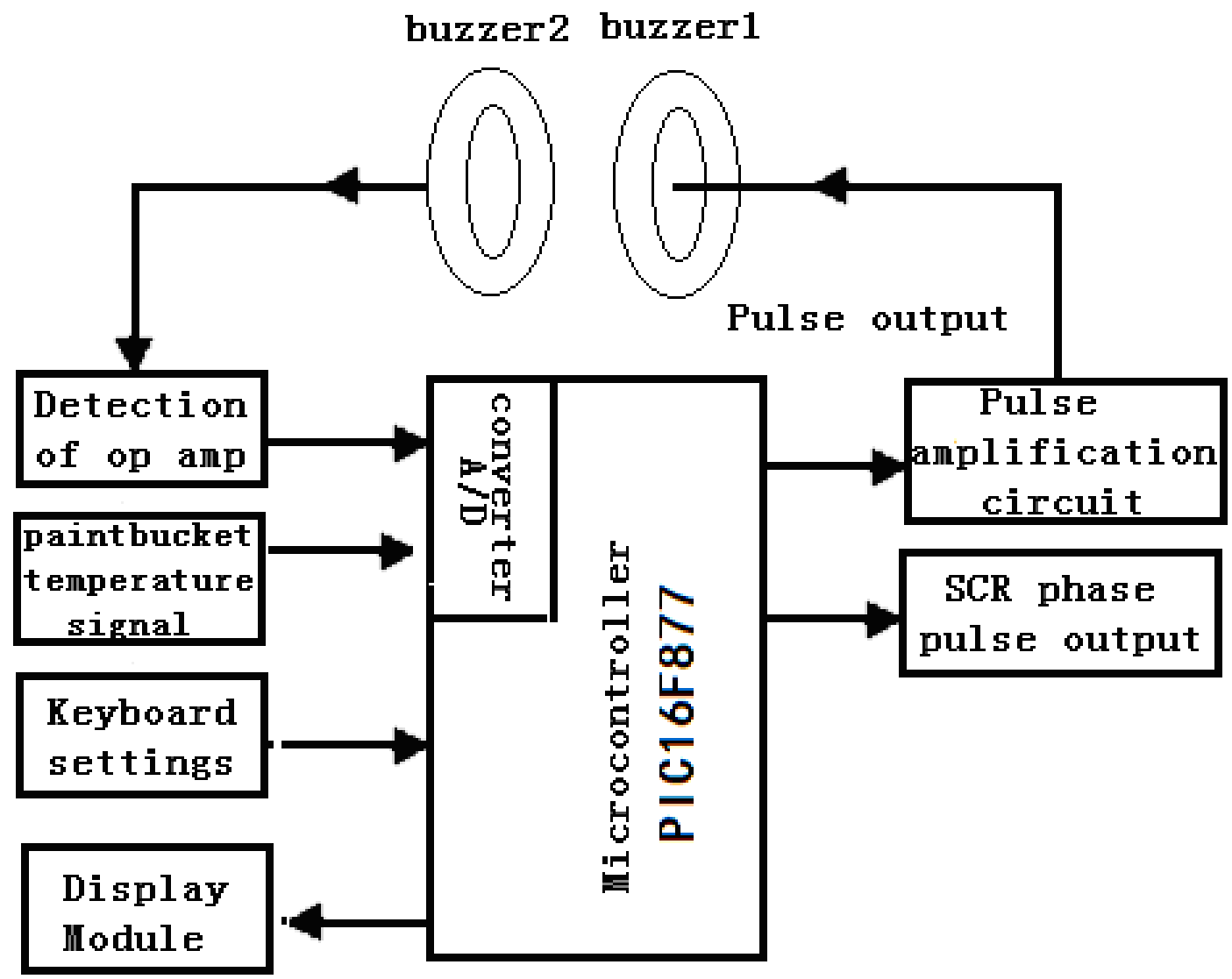

Fig. 1 Hardware circuit.

The Measurement Circuit of Piezoelectric Buzzer Charge. The electric charge amplifier makes up of the electric charge stage (high-pass filter), low pass filter (10 times amplifier), normalized amplifier, inverter output amplifier, overload indicator and stabilized voltage supply. The whole block diagram as shown in Fig. 2: 


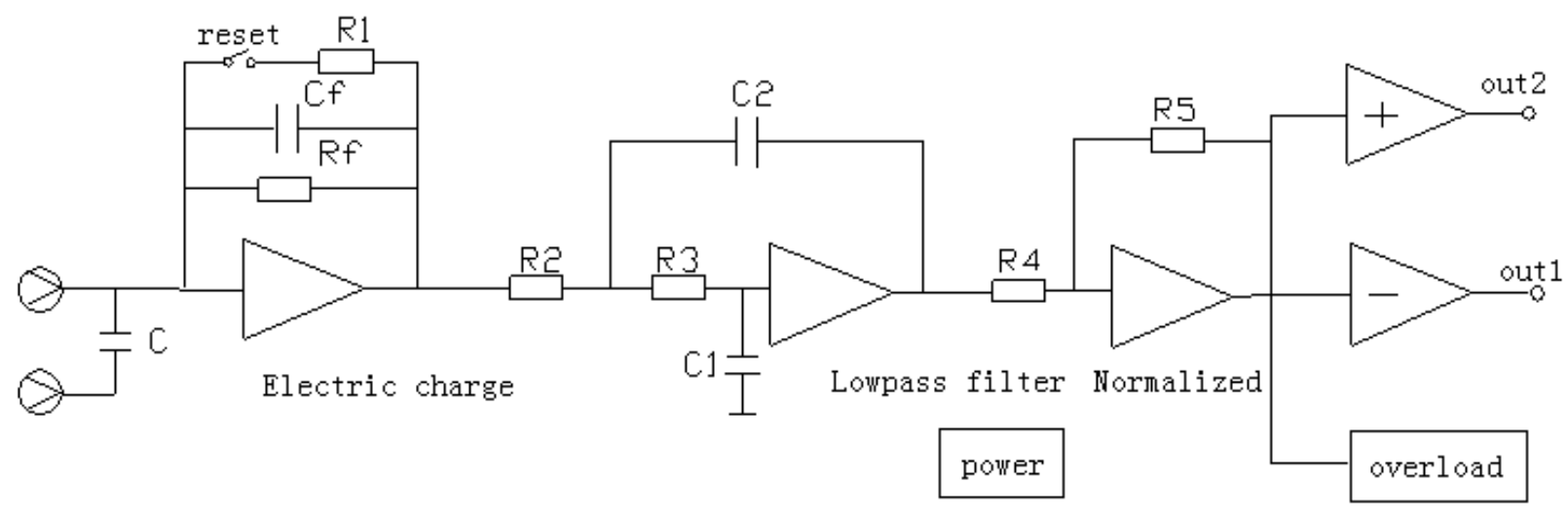

Fig. 2 measurement circuit of charge.

The stage of charge amplifier is composed by a pair of MOSFET field-effect tube and a high gain operational amplifier. The high impedance and low input current is used to calibrate the quasi static state and measure the extremely low frequency.

The lower frequency is obtained by conversion of different feedback resistance $\mathrm{R}_{\mathrm{f}}$, after sering between relay and resistor $\mathrm{R} 1$, and according to the feedback capacitance $\mathrm{C}_{\mathrm{f}}$, if the charge level is overloaded, the relay will gather and the circuit will reset rapidly. The low-pass filter is made up of operational amplifier R2, R3 and $\mathrm{C} 1$ and $\mathrm{C} 2$, the second-order active Bert water filter, we takes R2 $=\mathrm{R} 3 . \mathrm{C} 1=\mathrm{C} 2$, at the same time, winning six different upper limit frequencies through changing the $\mathrm{R} 2$ and R3. Inverter is an inverse proportion amplifier, takes $\mathrm{R} 4=\mathrm{R} 5$, making the input equals to the output. The output amplifier is an adjustable operational amplifier, which will give different gain corresponding to the different sensitivity of the sensor.

The Control Chip PIC16F877. Chip PIC16F877 has a high quality of performance, crystals work at the speed of $20 \mathrm{MHZ}$, has 8 depth hardware stacks, can address directly, indirectly and relatively, In addition to 35 single word transfer instructions, all the other instructions are all single cycle instruction which can calculate time conveniently. At the same time, the chip has an energy saving sleep mode and a durable flash memory EEPROM whose flash can write countless times, and there is a weak pull-up function on the programmable PORTB. The MCU PIC16F877 has TMR0, TMR1, TMR2, I ${ }^{2} \mathrm{C}$, SPI modules, synchronous and asynchronous serial port module, capture module, Trap module and PWM module.

\section{The Software Chart and the Main Program}

In this part, we will introduce the function of each module in the program flow chart, system initialization, the register makes initialize parameters setting, button setting: when the keyboard is pressed, the program will fall into the keyboard handler ones, changing the various setting parameters, return when the keyboard handed; Read the set data; take the temperature of the paint bucket to the A/D converter, adjust the trigger angle of the controlled silicon to make the paint of the paint bucket a constant temperature to measure the constant temperature conveniently ; When processed and conversed by the A/D converter, the piezoelectric signal of Piezoelectric buzzer will be sent to the LED display module as the mode of $\mathrm{I}^{2} \mathrm{C}$ to tell the workers the viscosity of paint. The program block diagram as shown in Fig. 3 . 


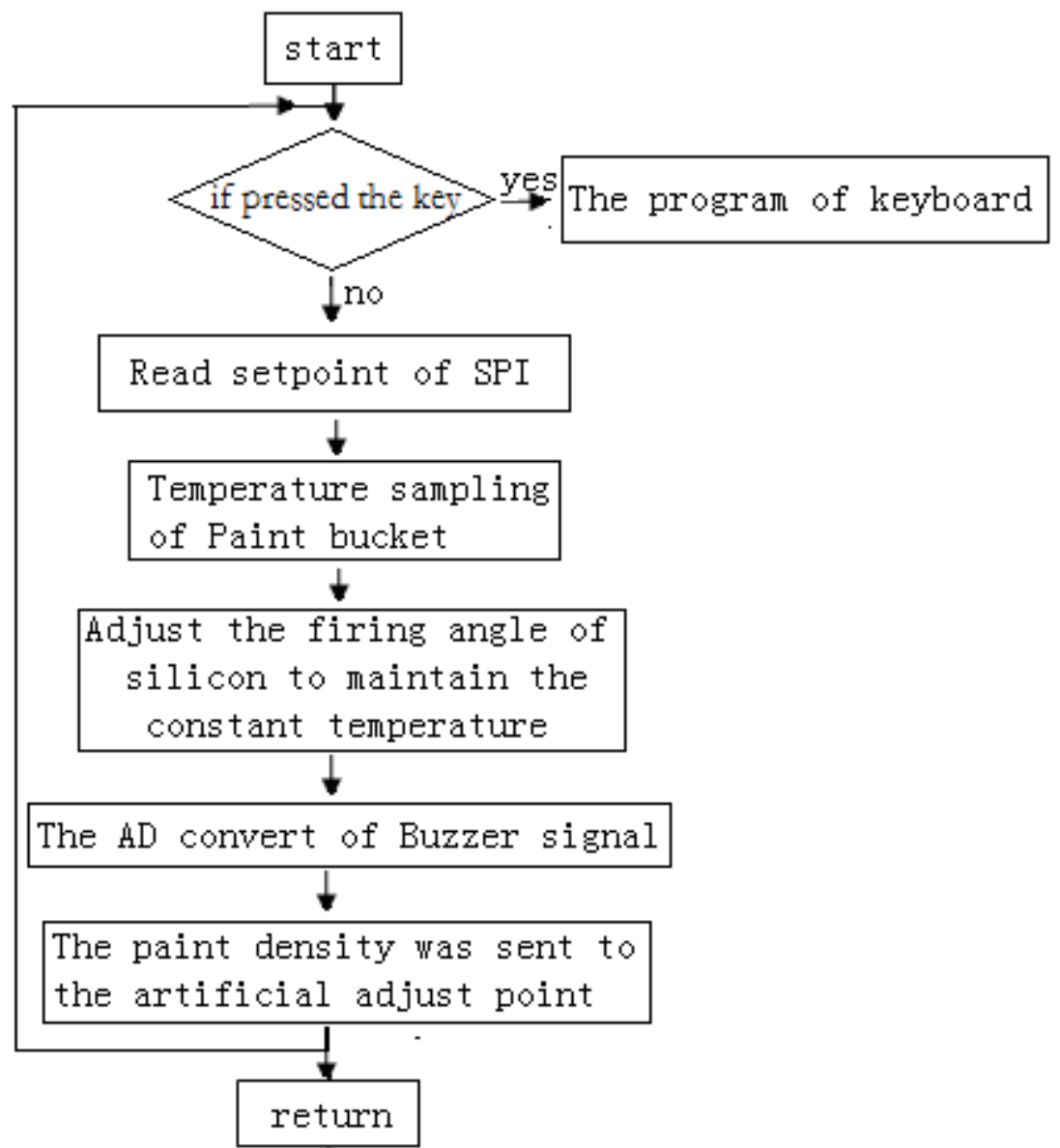

Fig. 3 The major software program flowchart.

Applicate $\mathrm{C}$ language to program, according to the scope the register uses in the program, defines a global variable and a local variable reasonably to save memory space. In the program, we use a switch sentence to achieve the function of keys, write the program distinctly, simply and clearly. And make sure not to lose the parameters of EEPROM .

void Delay MS(uint16 delay)// Delay function

\{

uint16 i; // Local variables

for(;delay $>0$;delay--)// Outer loop

for $(\mathrm{i}=0 ; \mathrm{i}<61 ; \mathrm{i}++)$; // Inner loop

\}

void Ml2035_Hz(uint16 hz)// Function name

\{

lati $=0 ; \quad$ // low Data line

sck $=0 ; \quad$ // Low clock line

ml_temp = hz \& 0x0001; // Save Low data

for $\left(m l \_t e m p==1\right) \quad$ //judgemnet of 1

sdi $=1 ; \quad$ // pull Data line

sck $=1 ; \quad$ // pull clock line

for $\left(\mathrm{ml} \_\mathrm{i}=0 ; \mathrm{ml} \_\mathrm{i}<15 ; \mathrm{ml} \_\mathrm{i}++\right)$

$\{$ sck $=0$;

$\mathrm{hz}=\mathrm{hz} \gg>1 ; \quad$ // Displacement

ml_temp $=$ hz \& 0x0001;

sck $=1 ; \quad$ // pull Data line

\}

sck $=0 ; \quad$ // Low clock line 


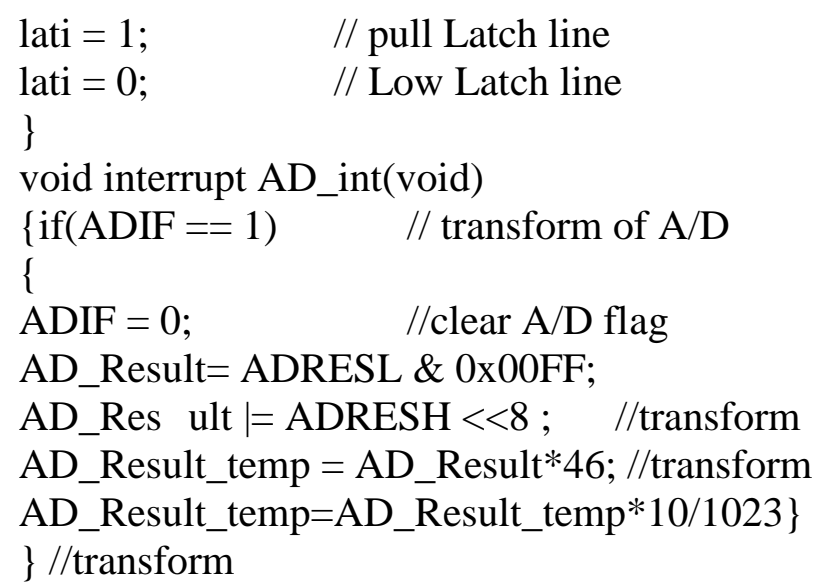

\section{Conclusion}

Enameled wire is an important, indispensable basic industrial material. In this passage, we described the piezoelectric effect and inverse piezoelectric effect of the Piezoelectric buzzer. In cooperation with PIC16F877 microcontroller, we designed the circuit and software, proposed a new method to measure the viscosity of paint dissolved liquid based on microcontroller and piezoelectric ceramic Piezoelectric buzzer piece, and solved the difficult problem in the process of producing. The whole design is simple and reasonable, reliable and economic. The application at the scene is very stable and the measured viscosity data is accurate.

\section{References}

[1] P. Hao, L. Zhang, J. L. Yu, Z. G. Xia, X. T. Li, Buzzer is the use of forced vibration measuring liquid viscosity, J. Phys. Experiments in 2006.

[2] Y. Li, The automatic package machine lacquer paint viscosity control system and precision analysis, the thesis. [meeting, - electric technology association, Jiangsu province, nanjing electric technology association, international society for electrical and electronics engineers (IEEE) Shanghai branch, 1999.

[3] M. Abdalla, J. H. An, M. Bellare, C. Namprempre, From identification to signatures via the Fiat-Shamir transform: Necessary and sufficient conditions for security and forward security, IEEE T. Inform. Theor. 54(8) (2008) 3631-3646.

[4] J. H. An, Y. Dodis, T. Rabin, On the security of joint signature and encryption, Adv. Cryptology-Eurocrypt, 2332 (2002) 83-107.

[5] B. Barak, M. Mahmoody-Ghidary. 2007. Lower bounds of signatures from symmetric primitives, In 48th Annal Symposium on Foundations of Computer Science, 290(1-2): 680-688.

[6] M. Bellare and S. Shoup, Two-tier signatures from the Fiat-Shamir transform with applications to strongly unforgeable and one-time signatures, IET Proc. Inform. Secur. 2(2) (2008) 47-63.

[7] D. Boneh, Short signatures without random oracles and the SDH assumption in bilinear groups, J. cryptol. 21(2) (2008) 149-177. 